\title{
The Globular Cluster System of NGC 5128: Ages, Metallicities, Kinematics and Structural Parameters
}

\author{
K. A. Woodley ${ }^{\mathrm{A}, \mathrm{C}, \mathrm{D}}$ and M. Gómez ${ }^{\mathrm{B}}$ \\ A Department of Physics \& Astronomy, McMaster University, Hamilton ON, L8S 4M1, Canada \\ B Departamento de Ciencias Fisicas, Facultad de Ingenieria, Universidad Andres Bello, Chile \\ ${ }^{\mathrm{C}}$ Department of Physics \& Astronomy, University of British Columbia, Vancouver BC, \\ V6T 1Z1, Canada \\ D Corresponding author. Email: kwoodley@phas.ubc.ca
}

Received 2009 September 19, accepted 2009 November 13

\begin{abstract}
We review our recent studies of the globular cluster system of NGC 5128. First, we have obtained low-resolution, high signal-to-noise spectroscopy of 72 globular clusters using Gemini-S/GMOS to obtain the ages, metallicities, and the level of alpha enrichment of the metal-poor and metal-rich globular cluster subpopulations. Second, we have explored the rotational signature and velocity dispersion of the galaxy's halo using over 560 globular clusters with radial velocity measurements. We have also compared the dependence of these properties on galactocentric distance and globular cluster age and metallicity. Using globular clusters as tracer objects, we have analyzed the mass, and mass-to-light ratio of NGC 5128. Last, we have measured the structural parameters, such as half-light radii, of over 570 globular clusters from a superb 1.2-squaredegree Magellan/IMACS image. We will present the findings of these studies and discuss the connection to the formation and evolution of NGC 5128.
\end{abstract}

Keywords: galaxies: elliptical and lenticular, $\mathrm{cD}$ - galaxies: evolution — galaxies: individual (NGC 5128) — galaxies: star clusters — globular clusters: general

\section{Introduction}

Globular clusters (GCs) survive for a Hubble time and undergo dissipationless dynamical evolution. They have been shown to form during major episodes of star formation (Holtzman et al. 1992; Schweizer \& Seitzer 1993, 1998; Whitmore et al. 1993; Whitmore \& Schweizer 1995; Zepf et al. 1995; Schweizer et al. 1996; Miller et al. 1997; Carlson et al. 1998; Whitmore et al. 1999; Zepf et al. 1999; Chien et al. 2007; Goudfrooij et al. 2007; Trancho et al. 2007, among others). This enables the use of GCs as probes of the formation history in their host galaxy which can tell us important information on the galaxy's assembly history and the physical conditions in which the GCs were formed. We can use their ages and metallicities to reconstruct the star formation history of their galaxy (West et al. 2004) as well as use the GCs as kinematic and dynamic tracers (Bridges et al. 2006).

There are many advantages in using GCs as probes of star formation and galaxy assembly. GCs are coeval and form with a single age and a single metallicity to first approximation. We also find large GC systems in early-type galaxies, providing a large basis for study. GC systems have been shown to exhibit two colour modes, both blue and red (Peng et al. 2006), indicating there must have been at least two episodes of star formation within these galaxies. Since the large majority of the colour change of the GC light that we see happens within the first few Gyr (Worthey et al. 1994), any subsequent colour difference is due to a difference in metallicity, so we refer to blue as metal-poor and red as metal-rich GCs. The advancements of multi-object spectrographs have allowed us to obtain numerous GC spectra in a homogeneous manner, providing large sample sizes.

\subsection{The Globular Cluster System of NGC 5128}

NGC 5128 is the nearest available giant elliptical for study at $3.8 \pm 0.1 \mathrm{Mpc}$ (G. L. H Harris, 2009, private communication). Its close proximity provides the opportunity to perform a detailed study of its GC system at a level of detail that is not possible in other giant ellipticals at present. We will use the GCs as representations of the bulk stellar population to probe its formation history.

In NGC 5128, there are an estimated $1500 \mathrm{GCs}$ within 25 arcmin (Harris et al. 2006). We currently know of 70 GCs within NGC 5128 that have been identified as resolved GCs from Hubble Space Telescope images (Harris et al. 2006). There are 564 GCs also confirmed by radial velocity measurement (van den Bergh, Hesser \& Harris 1981; Hesser et al. 1984; Hesser, Harris \& Harris 1986; Harris et al. 1992; Peng et al. 2004b; Woodley et al. 2005; Rejkuba et al. 2007; Beasley et al. 2008), including 190 new GCs our group has recently identified (Woodley et al. 2009, 2010). The sample now includes 605 GCs which is one of the largest samples of GCs in the literature. Of the total GC system with known photometry, we have identified 268 metal-poor and 271 metal-rich 


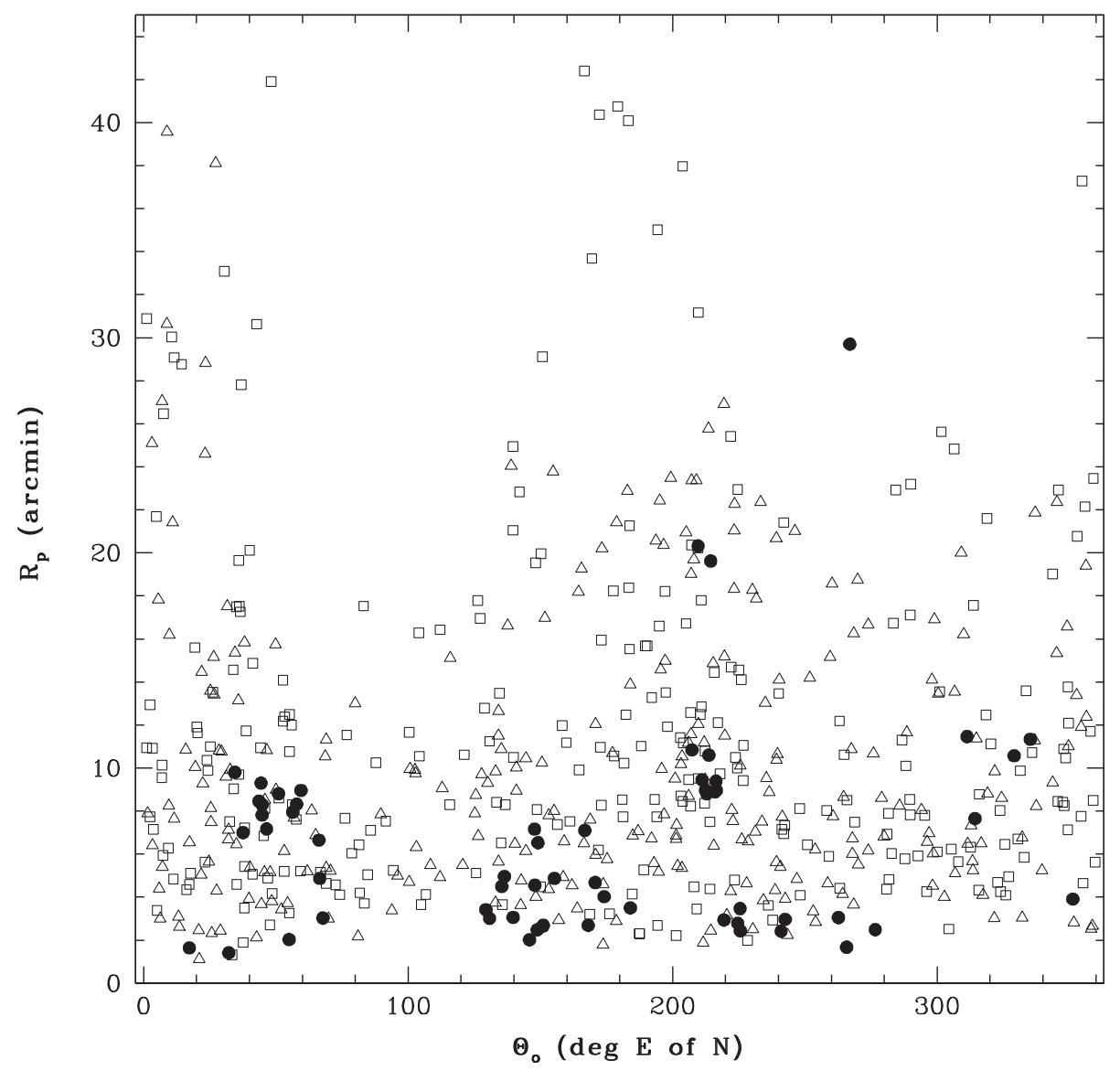

Figure 1 The galactocentric radial distribution of GCs in NGC 5128 as function of azimuthal position, measured in degrees E of N. The squares and the triangles are the metal-poor and metal-rich GCs with a measured velocity, respectively, and the solid circles are GCs that either have measured velocities but no colour information, or GCs with no measured radial velocities.

GCs, with a clear bimodal distribution in colour. Figure 1 shows the projected spatial distribution of the GC system which extends out to 45 arcmin in galactocentric radius, distributed mainly along the isophotal major axis of the galaxy and heavily concentrated within 15 arcmin. Our sample is thus spatially biased, caused primarily by the chosen field locations of previous studies.

\section{The Ages, Metallicities and Alpha-Enhancement of the Globular Clusters}

\subsection{Index Measurement}

To obtain ages and metallicities of the GCs in NGC 5128 , we need to obtain high signal-to-noise $(\mathrm{S} / \mathrm{N})$ spectroscopy to measure the strength of particular absorption features. In NGC 5128, we have obtained integrated light spectroscopy of GCs with Gemini-S/GMOS. Our field placements are centrally concentrated within 15 arcmin, but distributed azimuthally. We obtained spectra for 72 GCs with $\mathrm{S} / \mathrm{N}>30$ covering the wavelength regime of 3800-5500 ̊. The measured indices include the Balmer lines, as well as $\mathrm{Mg}, \mathrm{CN}$ and many Fe lines. The data reduction details are described in Woodley et al. (2010).

We measured the indices using GONZO (Puzia et al. 2002) and calibrated the data to the Lick Index System (described in Burstein et al. 1984; Worthey et al.
1994; Worthey \& Ottaviani 1997; Trager et al. 1998), a standardized index system. We had between 9 and 16 GCs in common with Beasley et al. (2008) who had directly calibrated their data to the Lick index system via standard stars. With Lick indices in hand, we iterated between our measured indices and the simple stellar population model grids of Thomas, Maraston \& Bender (2003) and Thomas, Maraston \& Bender (2004) to obtain ages, metallicities, and $[\alpha / \mathrm{Fe}]$ of the GCs. We have performed the same analysis with integrated light spectra of 40 Milky Way GCs from Schiavon et al. (2005) and 1 Milky Way GC from Puzia et al. (2002). We calibrated the Milky Way GC to the Lick system via 11 GCs in common with Puzia et al. (2002), who have calibrated their data to the Lick system with standard stars. The uncertainties in our measured indices are determined using GONZO by adding Poisson noise to each input GC spectra via 100 Montecarlo simulations. The uncertainty on each index is the $1 \sigma$ standard deviation of the index measurement. The list of indices with uncertainties, as well as the various calibration plots are shown in Woodley et al. (2010).

\subsection{Spectroscopic Results and Discussion}

We show an example of one diagnostic plot in Figure 2. Here we have shown the GC indices for both NGC 5128 


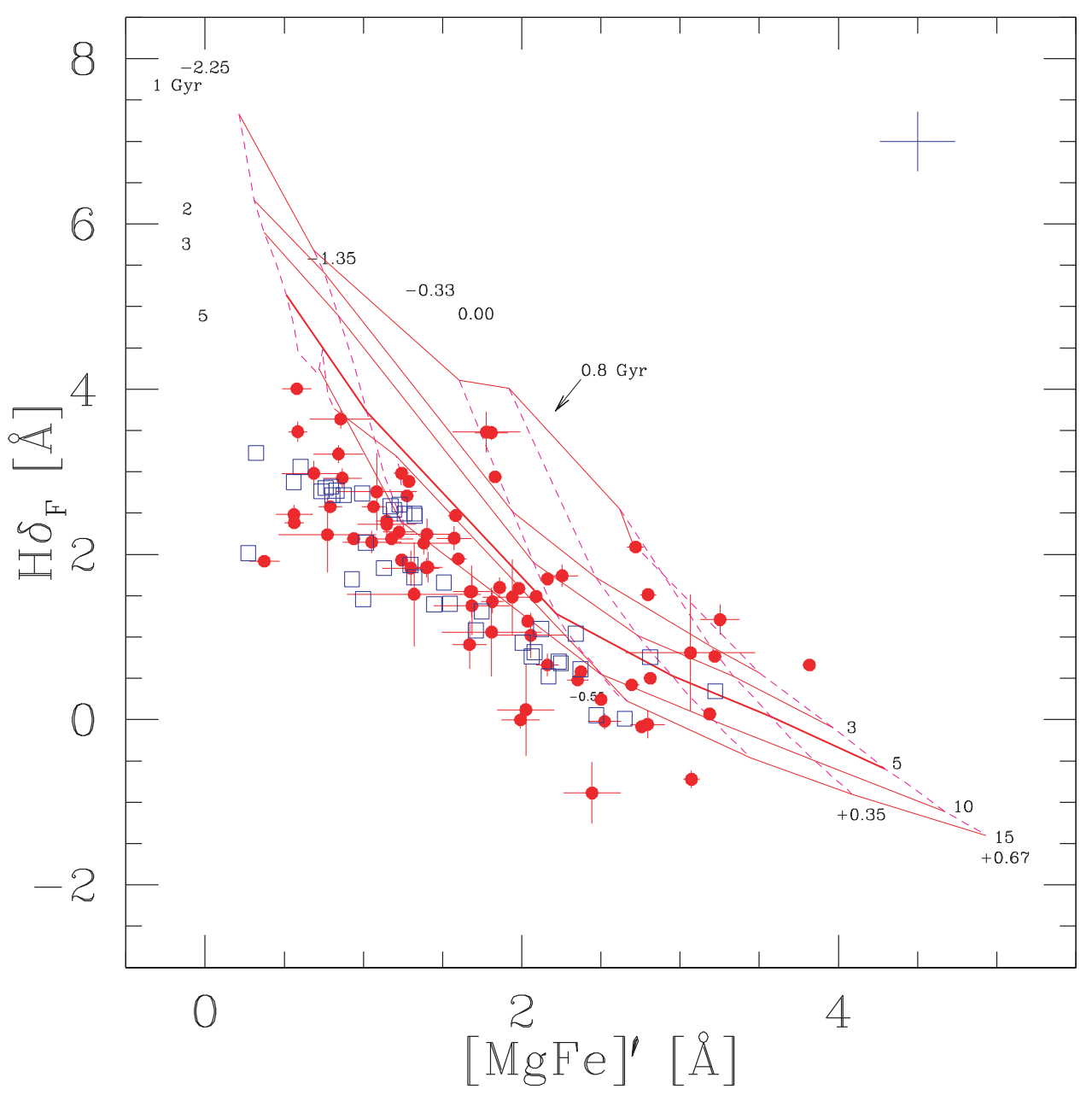

Figure 2 The measured indices for 72 GCs in NGC 5128 (red circles) and 41 GCs in the Milky Way (blue squares) shown for $\mathrm{H} \delta_{F}$ and $[\mathrm{MgFe}]^{\prime}$. The systemic uncertainty is shown in the upper right (blue cross). Overplotted are the simple stellar population models of Thomas et al. (2003, 2004). This figure is taken from Woodley et al. (2010).

and the Milky Way plotted for the higher order Balmer line $\mathrm{H} \delta_{F}$ vs. $[\mathrm{MgFe}]^{\prime 1}$, sensitive to age and metallicity, respectively. Overplotted are the simple stellar population models of Thomas et al. $(2003,2004)$ with an $[\alpha / \mathrm{Fe}]$ of solar. To obtain age, metallicity, and $[\alpha / \mathrm{Fe}]$ for the GCs, we use an iteration technique incorporating a number of diagnostic plots, including the Balmer lines, $\mathrm{Mg}_{2}, \mathrm{Mg}_{b}$, Fe5270 and Fe5335 with varying $[\alpha / \mathrm{Fe}]$ (described in full in Puzia et al. 2002). Our results are presented in Figure 3. The histrograms have been fit with Gaussian distributions using the statistical code RMIX ${ }^{2}$.

We clearly see a difference in the age distribution functions between the GCs in NGC 5128 and those in the Milky Way. A Kolmogorov-Smirnov statistical comparison test indicates these two distributions are different at greater than a 99\% confidence level. There appears to be multiple epochs for formation history in NGC 5128. An important test of our technique is the ability to reproduce the known

\footnotetext{
${ }^{1}[\mathrm{MgFe}]^{\prime}=\left[\mathrm{Mg}_{b} \times(0.72 \times \mathrm{Fe} 5270+0.28 \times \mathrm{Fe} 5335)\right]^{1 / 2} \quad$ (Thomas et al. 2003)

2 The complete code, available for a variety of platforms, is publicly available from Peter MacDonald's Web site at http: //www. math. mcmaster.ca/peter/mix/mix.html.
}

old ages of the Milky Way GCs. We have obtained a mean age of $11.3 \pm 0.1$ Gyr for the Milky Way GCs, in excellent agreement with the known old ages of the Milky Way GCs. We have confidence therefore in our own results for the GCs in NGC 5128. We find, in NGC 5128, $68 \%$ of our GC sample have old ages greater than $8 \mathrm{Gyr}, 14 \%$ have intermediate ages between 5 and $8 \mathrm{Gyr}$, and $18 \%$ have young ages less than 5 Gyr.

The metallicity distribution, determined using spectroscopic indices with the simple stellar population models, provides a distribution that is not clearly bimodal, as seen in the Milky Way. Our sample size is quite small and is heavily biased towards metal-rich as well as younger GCs because we are specifically targetting the central region of NGC 5128. In this region, the metal-rich population is more strongly concentrated than the metal-poor (see Woodley et al. 2005, 2007; Beasley et al. 2008). We are also more likely to target younger objects, which are brighter than their older counterparts, to achieve the required $\mathrm{S} / \mathrm{N}$. We also have a bias towards a larger range of ages than perhaps the outer halo, due to accreted material sinking towards the central regions. Our proportion of young, metal-rich GCs is therefore, likely inflated. We 

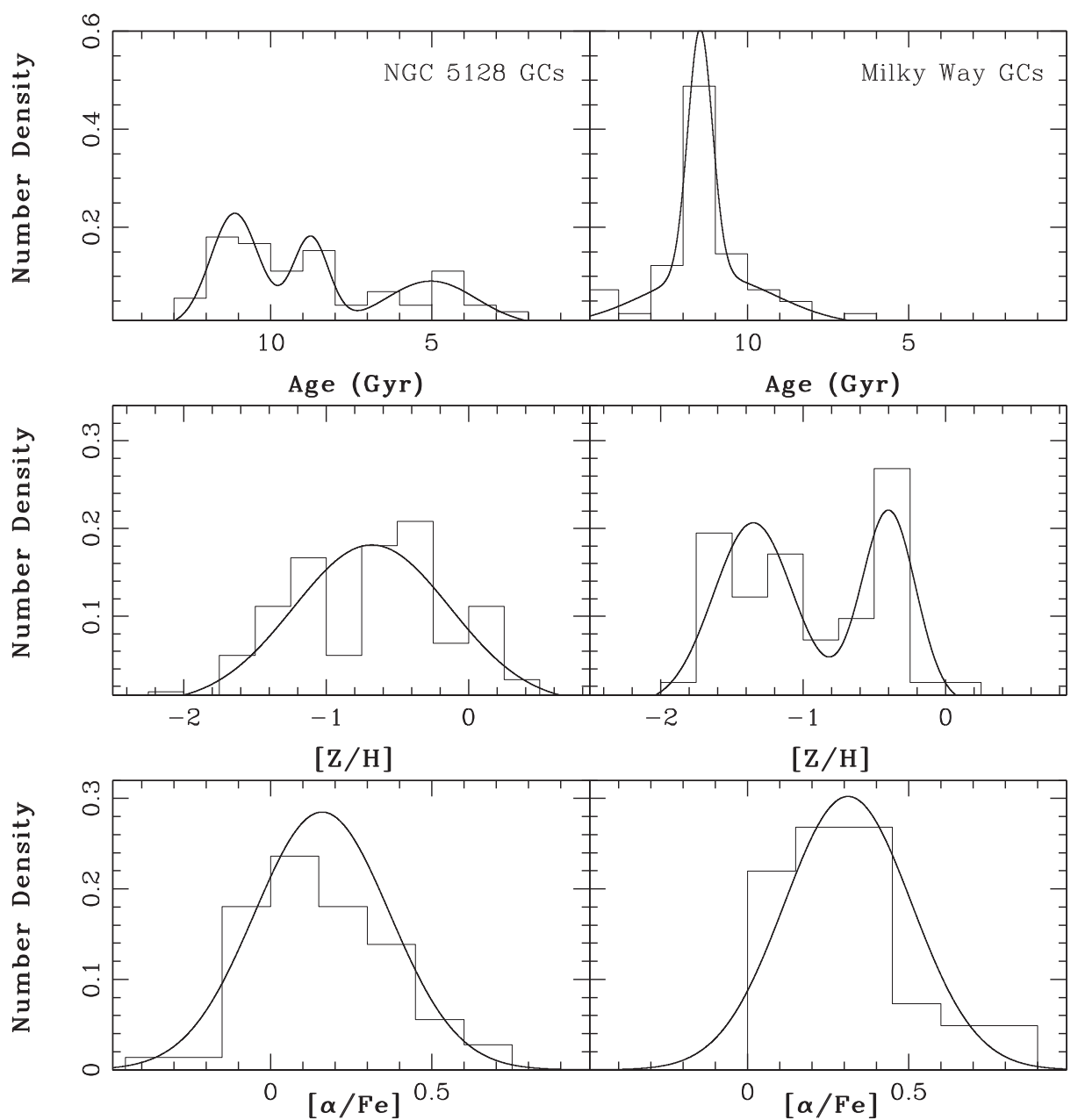

Figure 3 The age (top), metallicity (middle), and $[\alpha / \mathrm{Fe}]$ (bottom) distributions for the GCs in NGC 5128 (left) and the Milky Way (right). The best fit Gaussians are plotted. This figure has been modified from Woodley et al. (2010).

find that that $92 \%(23 / 25)$ of metal-poor GCs and $56 \%$ (26/47) of metal-rich GCs that we have sampled in NGC 5128 have ages $>8 \mathrm{Gyr}$, consistent with the old ages of the Milky Way GCs. We do however find all younger GCs are metal-rich, with the exception of 2 metal-poor GC with an intermediate age.

Lastly, we obtain the abundance of $\alpha$-to-Fe type elements. This ratio can indicate the timescale of GC formation. Supernovae type II events occur over a timescale of $\sim 100 \mathrm{Myr}$ and enrich the interstellar medium with primarily $\alpha$-type elements. The onset of supernova type Ia events, which enriches the interstellar medium with a larger fraction of Fe-type elements after $1 \mathrm{Gyr}$, will reduce the value of $[\alpha / \mathrm{Fe}]$. In NGC 5128, we find a spread in $[\alpha / \mathrm{Fe}]$ at every given metallicity and a wider spread among the older GCs. The mean $[\alpha / \mathrm{Fe}]$ for the GCs in NGC 5128 is $0.14 \pm 0.04$ dex which indicates a fast formation timescale, likely forming in a rapid cloud collapse, as opposed to a merger event, which likely enrich the GCs to solar $[\alpha / \mathrm{Fe}]$.

\section{Kinematics of the Globular Cluster System}

The bimodality of the GC system suggests there may be multiple epochs of star formation. This could also lead to different kinematic signatures between the metal-rich and metal-poor GCs. We explore the kinematics signature of the 564 GCs with radial velocity measurements by fitting the standard sine curve fit to the data,

$$
v_{\mathrm{p}}(\Theta)=v_{\mathrm{sys}}+\Omega R \sin \left(\Theta-\Theta_{\mathrm{o}}\right)
$$

described in Côté et al. (2001). Our input values are the measured radial velocity, $v_{\mathrm{p}}$, and the angular position of each GC measured on the projected sky in degrees east of north, $\Theta$. The radial velocities and associated uncertainties used in this study are the weighted averages of all previous measurements in the literature. We extract the systemic velocity, $v_{\mathrm{sys}}$, the rotation amplitude, $\Omega R$, and the projected rotation axis, $\Theta_{0}$, also measured in degrees East of North in the projected sky, with a weighted least squares non-linear fit.

We bin the data in two ways. The first binning method is by radial position in the galaxy so the bins are independent and plotted at the mean radial value of all GCs in that bin. The second binning method is an exponentially weighted binning technique described in Bergond et al. (2006). 
Table 1. Kinematics of the GC system

\begin{tabular}{lcc}
\hline & Metal-rich & Metal-poor \\
\hline$v_{\mathrm{sys}}\left(\mathrm{km} \mathrm{s}^{-1}\right)$ & $527 \pm 10$ & $504 \pm 10$ \\
$\Omega R\left(\mathrm{~km} \mathrm{~s}^{-1}\right)$ & $41 \pm 15$ & $17 \pm 14$ \\
$\Theta_{\mathrm{o}}(\mathrm{deg} \mathrm{E} \text { of } \mathrm{N})_{\sigma_{\mathrm{p}}}\left(\mathrm{km} \mathrm{s}^{-1}\right)$ & $191 \pm 18$ & $154 \pm 47$ \\
\hline
\end{tabular}

Table 2. Kinematics of the age groups of GCs

\begin{tabular}{lrrr}
\hline & $>8 \mathrm{Gyr}$ & $5-8 \mathrm{Gyr}$ & $<5 \mathrm{Gyr}$ \\
\hline$v_{\mathrm{sys}}\left(\mathrm{km} \mathrm{s}^{-1}\right)$ & $543 \pm 23$ & $577 \pm 42$ & $562 \pm 51$ \\
$\Omega R\left(\mathrm{~km} \mathrm{~s}^{-1}\right)$ & $27 \pm 36$ & $53 \pm 78$ & $58 \pm 92$ \\
$\Theta_{\mathrm{o}}(\operatorname{deg~E~of~N)}$ & $107 \pm 65$ & $235 \pm 54$ & $80 \pm 84$
\end{tabular}

\subsection{Kinematic Results}

While we present only selected results here, the full results can be found in Woodley et al. (2009). Table 1 lists the $v_{\mathrm{sys}}$, $\Omega R, \Theta_{\mathrm{o}}$, and the velocity dispersion, $\sigma_{\mathrm{v}_{\mathrm{p}}}$, for the metal-rich and metal-poor GC samples determined with Equation 1.

We clearly see, as a whole, the GC system is not strongly rotating and is thus supported by random motion. The metal-rich GCs appear to have mild rotation around an axis similar to the isophotal major axis of the galaxy, located at $35^{\circ}$ and $215^{\circ} \mathrm{E}$ of $\mathrm{N}$ (Dufour et al. 1979) in the inner 15 arcmin. However, the metal-poor GCs with very mild rotation, do not appear to rotate around either the major or minor isophotal axis. The velocity dispersion is also determined for both the metal-rich and metal-poor GC subpopulations, and are identical within uncertainties. This is also seen in the GC system of the elliptical NGC 4636 (Schuberth et al. 2006).

We examine the metal-rich GC population rotation axis more closely by binning the data in independent galactocentric bins as well as by an exponential fitting, shown in Figure 4. For the metal-rich GCs, the bin widths are $0-5,5-10,10-15$ and $15-45$ arcmin. The rotation axis of the metal-rich GCs does rotate around an axis near the isophotal major axis, at least within the inner 10 arcmin of the galaxy. We also examine the kinematics for the age subsamples determined in Section 2, and are summarized in Table 2. We note the rotation axis of the metal-rich GCs with ages $<5 \mathrm{Gyr}$, do not rotate around a similar axis as the bulk of old, metal-rich GCs. The uncertainties are quite large because of the small number of young GCs in our sample $(N=13)$, but it could indicate the kinematics among the youngest GCs are different than the bulk of the GC system. A larger sample of GC ages are needed to examine this more closely.

We also examine the velocity dispersion for the entire GC system, as a function of radius, shown in Figure 5. In this plot, the radial bins are $0-5,5-10,10-15,15-20$ and
20-45 arcmin for the entire GC system. The velocity dispersion appear flat within the inner 15 arcmin, after which it shows an increase. Although not shown in Figure 5, we note a similar velocity dispersion profile for both the metal-rich GC and metal-poor GC subpopulations separately (Woodley et al. 2009). This is also the onset of the spatial biases within the GC system and needs to be examined more closely with a larger, more azimuthally symmetric sample of GCs. With this caveat aside, an increasing velocity dispersion in the outer regions could be interpreted as the galaxy containing a large amount of dark matter or the GCs are moving on anisotropic orbits. In NGC 5128 it is difficult to analyze the orbital motion of the GCs without modelling and/or without an external mass estimate extending to these outer regions. Similar findings have been noted in M87 (Côté et al. 2001), as well as hints for an increase in the velocity dispersion of the GCs in the elliptical galaxies NGC 3379 (Pierce et al. 2006) and M49 (Côté et al. 2003).

\subsection{Comparison to the Planetary Nebulae and the Stellar Halo}

The planetary nebulae (PNe) are the most direct look at the field star population in NGC 5128. There is a sample of 780 PNe in NGC 5128 (Hui et al. 1995; Peng et al. 2004a) extending out to $\sim 80$ arcmin from the centre of the galaxy, strongly concentrated along the well search isophotal major axis. We determine the rotation axis of the PNe and show its profile distribution binned radially in Figure 4. Similar to the metal-rich GCs, it rotates around an axis near the isophotal major axis. We compare the PNe velocity dispersion to that of the GC system in Figure 5 and see a difference between the two systems. The bin sizes for the PNe data are 0-5, 5-10, 10-15, 15-20, 20-25 and 25-80 arcmin. The PNe profile appears to decrease and flatten near 10-15 arcmin, and then decrease out to beyond 30 arcmin, where the GC velocity dispersion appears to increase. The decrease in velocity dispersion of the planetary nebuale could indicate that the PNe are on anisotropic orbits. This behaviour has been seen in simulations of merging galaxies (for e.g. Dekel et al. 2005) where the PNe get thrown off their orbits from the disks of their progenitor galaxies into the halo of the merger remnant. We also note that the metal-rich GCs and the PNe have very similar surface density profiles, with slopes of $3.56 \pm 0.21$ and $3.47 \pm 0.12$ respectively, analyzed between 5 and 20 arcmin, where we are most secure with both sample populations. Further comparison with metallicity distribution of the stellar halo population generated from Hubble Space Telescope data (Rejkuba et al. 2005, and references therein), shows a similar enrichment to the metal-rich GCs. This is shown graphically in Woodley et al. (2010). Rejkuba et al. (2005) also estimate an age for the stellar halo population of $\sim 8_{-3.5}^{+3} \mathrm{Gyr}$, indicating the stellar population, along with the results shown here for the metal-rich GCs, are old. These increasing similarities between the metal-rich GCs and the stellar halo 


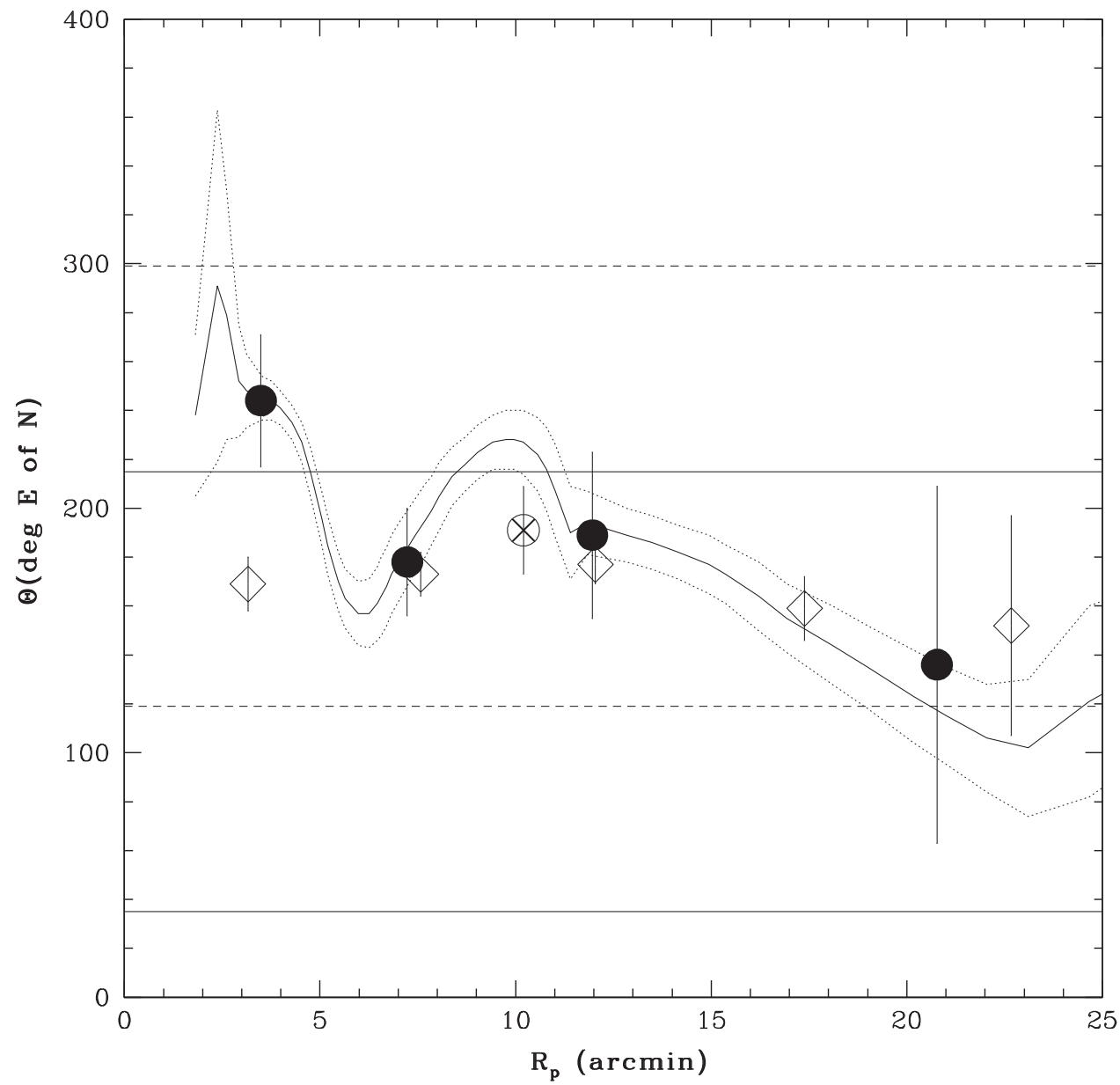

Figure 4 The rotation axis as a function of galactocentric radius for the metal-rich GCs binned radially (circles) and exponentially (curve), and for the PNe (diamonds). The results for the entire metal-rich population (circle with $x$ ). The isophotal major (solid lines) and minor (dashed lines) axes are shown.

population could indicate that they formed contemporaneously. However, we cautiously note the velocity dispersion profiles appear to differ between the metal-rich GCs and the PNe.

\subsection{Mass and Mass-to-Light of NGC 5128}

GCs can also be used to trace the mass of its host galaxy. Using our sample of 564 GCs with radial velocity measurements, we use the GCs as tracer particles to determine a total mass of the galaxy. The total mass is comprised of the rotationally supported mass added to the pressure supported mass. We determine the rotational component of the mass with the spherical Jeans equation and the pressure supported mass with the Tracer Mass Estimator (Evans et al. 2003).

Using the GCs that reside in the least spatially biased regime (between 5 and $20 \mathrm{arcmin}$ ), we estimate a mass of NGC 5128 of $5.5 \pm 1.9 \times 10^{11} \mathrm{M}_{\odot}$ and a mass-to-light ratio of $15.35 M_{\odot} / L_{B \odot}$ (assuming $B=7.84$ for NGC 5128; Karachentsev et al. 2002) out to $20 \mathrm{arcmin}$. This mass estimate agrees quite well with other recent work (Schiminovish et al. 1994; Peng et al. 2004b; Woodley et al. 2007).

\subsection{Kinematic Discussion}

For the large GC kinematic analysis presented in the literature, there is a wide range of results. There are also no clear patterns among the giant ellipticals in the Virgo cluster of galaxies, M60 (Bridges et al. 2006; Hwang et al. 2008), M87 (Côté et al. 2001), M49 (Côté et al. 2003) and NGC 4636 (Schuberth et al. 2006), as well as no clear patterns between the giant elliptical galaxies near the dynamical centres of their cluster of galaxies M87 (Côté et al. 2001) and NGC 1399 (Richtler et al. 2004), or the brightest ellipticals in their host cluster of galaxies M49 (Côté et al. 2003) and NGC 1399 (Richtler et al. 2004).

Bekki et al. (2005) have numerically simulated the kinematic signatures of preexisting metal-rich and metalpoor GCs from the merging of galaxies like our Milky Way. While their simulations were dissipationless, we are interested in comparing their kinematic predictions to our findings, since the bulk of the GC population in NGC 5128 is old. In their simulations, they found increasing rotational signatures with increasing galactocentric radius from the merger, due to transfer to angular momentum into the outer regions of the remnant. We have analyzed the rotation amplitude of NGC 5128 for both the metalrich and metal-poor GCs separately, and do not see a 


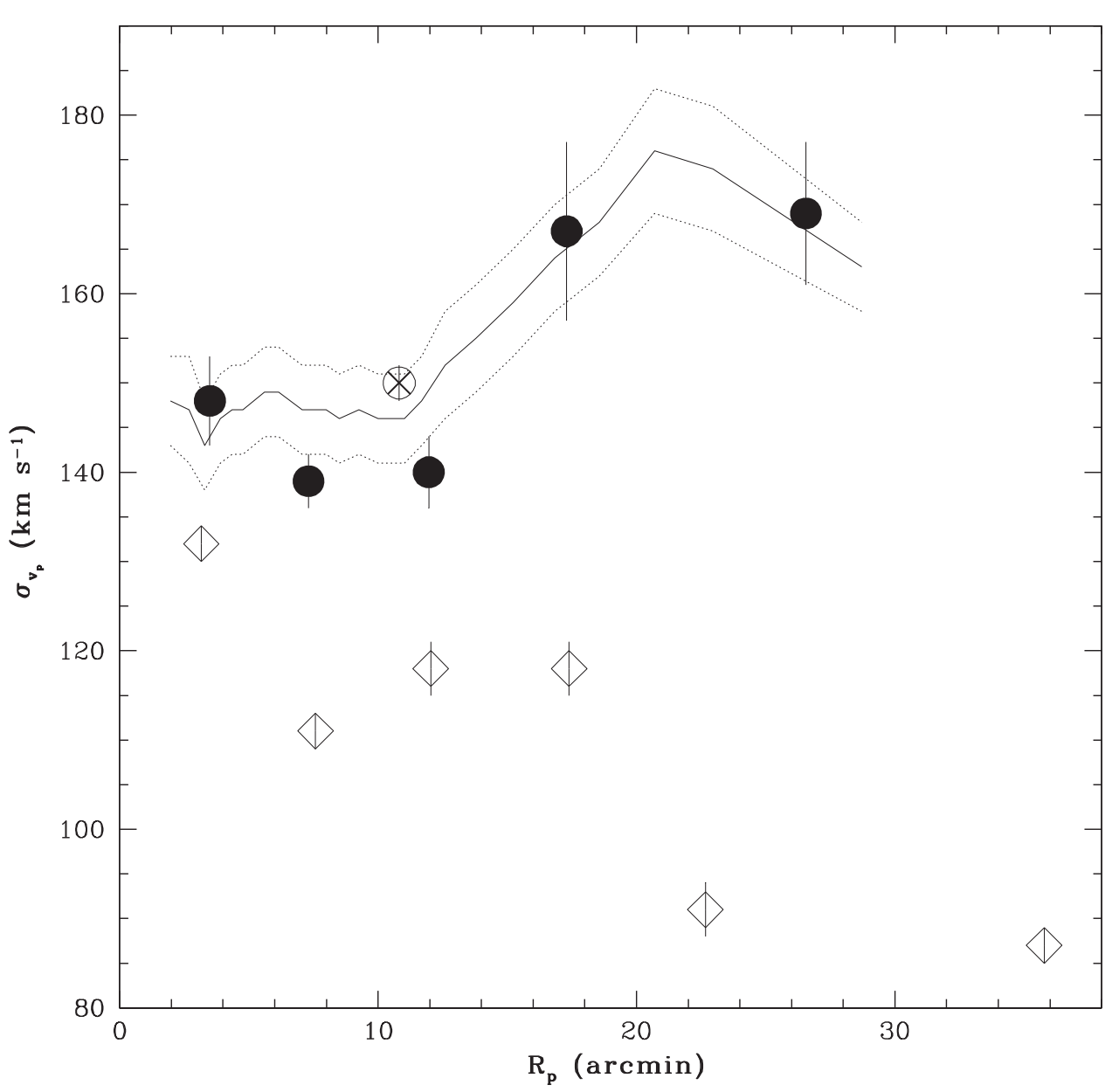

Figure 5 The velocity dispersion as a function of galactocentric radius for the entire GC system binned radially (circles) and exponentially (curve), and for the PNe (diamonds). We also show the results for the entire GC system (circle with $x$ ).

strong indication for increasing rotation with radius. The simulations of Bekki et al. (2005) also show the velocity dispersion for the GCs remained flat or decrease with radius. We see an evidence for a flat dispersion out to 15 arcmin from the centre. This comparison indicates that NGC 5128 may not be consistent with having formed as the result of the merger of two fully formed disks galaxies, although this is not ruled out.

An important implication from the study of Bekki et al. (2005) suggests that the initial kinematic signatures of GCs in the progenitor galaxies undergo orbital mixing. It may not be possible to trace the original kinematics of the GCs (Hwang et al. 2008), but only the GC kinematics from the most recent major interaction. If this were the case, it would be very difficult to use the current kinematics of the GCs to trace their orbital history (Kissler-Patig et al. 1998).

\section{Structural Parameters of the Globular Clusters}

The structural parameters of GCs can provide insight into how GCs form as well as their environment of formation. In particular, we are interested in their half-light radii, $r_{h}$. This parameter has been shown to remain relatively constant throughout the lifetime of a GC (Spitzer \& Thuan 1972; Aarseth et al. 1998). Interestingly, over the last decade, many studies have shown that the red GCs are smaller than the blue GCs by $17-30 \%$ in both spiral and elliptical galaxies (Kundu \& Whitmore 1998; Kundu et al. 1999; Puzia et al. 1999; Kundu \& Whitmore 2001; Larsen et al. 2001a; Larsen, Forbes \& Brodie 2001b; Barmby, Holland \& Huchra 2002; Jordán et al. 2005; Harris 2009). Almost all of these studies were performed with the small field of view of the Hubble Space Telescope, mainly centred on the inner regions of their respective galaxies. Spitler et al. (2006) examined the sizes of the GCs in the Sombrero galaxy (NGC 4594), using the Hubble Space Telescope, but extending to a few effective radii of the galaxy, $R_{\text {eff }}$. They showed that the red GCs were smaller than blue within the first $2 R_{\text {eff }}$. Beyond this distance their mean sizes did not appear to differ.

Our group has Magellan/IMACS images taken in 0.45 arcsec seeing that extend out to 40 arcmin in galactocentric radius. With this stunning image, we can measure all GCs with half-light diameters greater than $4 \mathrm{pc}$ ( 0.22 arcsec). In the Milky Way, this only misses $15 \%$ of the GCs. These IMACS images give us the opportunity to study the trends of the blue and red GCs homogeneously and extending to large radii.

We have measured the structural parameters with ISHAPE (Larsen 1999, 2001) which convolves the stellar 


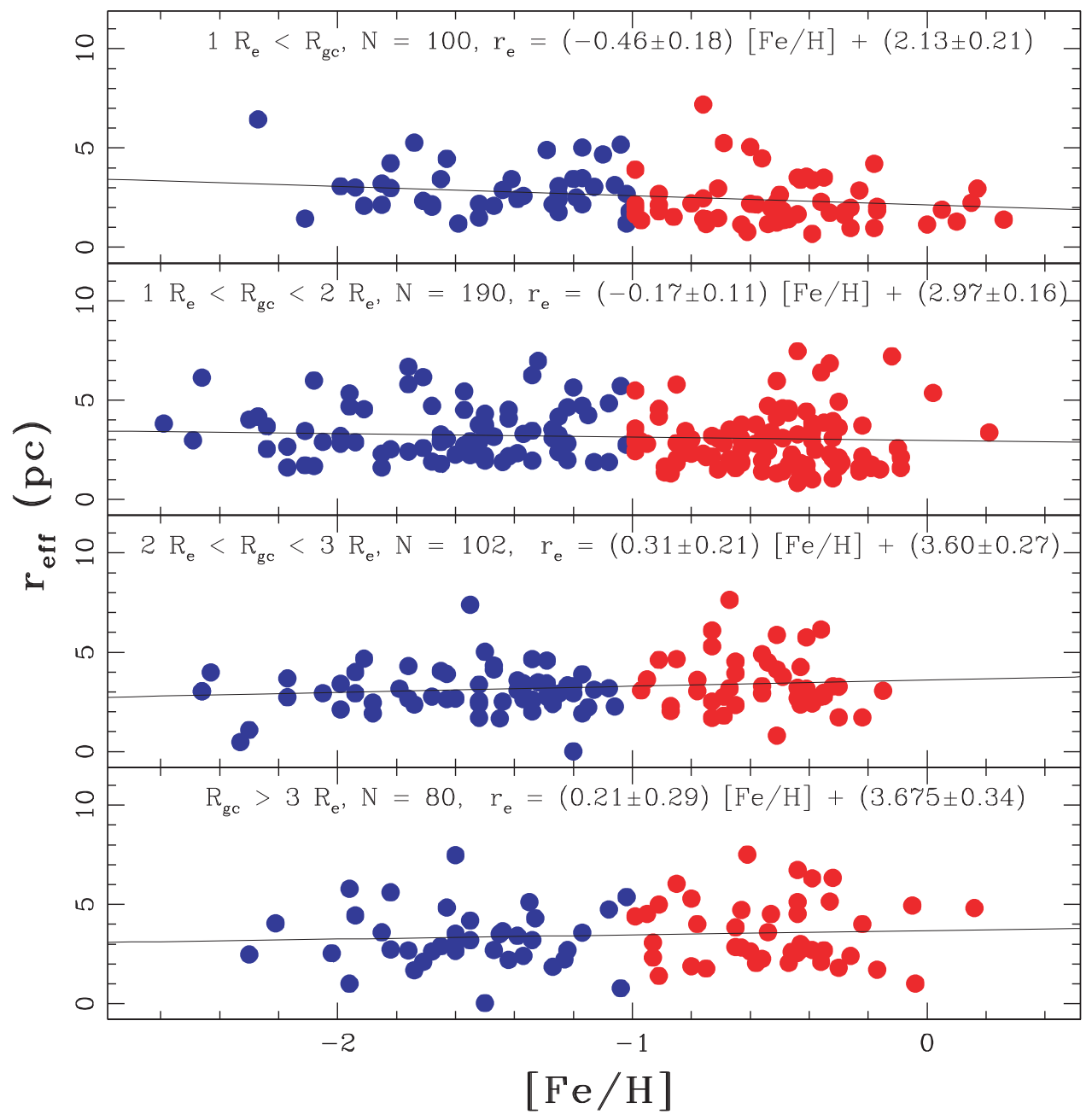

Figure 6 The half-light radius for the GC system binned in effective radius of the galaxy, increasing in galactocentric distance from the top to bottom panels, as a function of metallicity $[\mathrm{Fe} / \mathrm{H}]$. This is plotted for both blue and red GCs (shown as blue and red circles, respectively). The data in each bin has a least squares fit overplotted.

point spread function with an analytical King profile (King 1962) and compares the result with the input candidate image achieving a best match. The structural parameters, measured from the models, are the core radius, $r_{c}$, the tidal radius, $r_{t}$, the concentration parameter, $c=r_{t} / r_{c}$, and ellipticity. The half-light radii can also be obtained from the transformation, $r_{e} / r_{c} \simeq 0.547 c^{0.486}$ which is good to $\pm 2 \%$ for $c>4$ (Larsen 2001), which is satisfied for GCs in NGC 5128 (Goméz \& Woodley 2007). We measured the structural parameters for a total of 572 GCs in NGC 5128 and compare the results for both red and blue GCs. In this study, the metallicity, $[\mathrm{Fe} / \mathrm{H}]$, is a conversion from a $(\mathrm{C}$ $\mathrm{T}_{1}$ ) colour relation, derived by Harris \& Harris (2002) calibrated through the Milky Way GC data. We use a foreground reddening value of $E(B-V)=0.11$ for NGC 5128 in this relation. When there was no available $C$ $\mathrm{T}_{1}$ colour information for a $\mathrm{GC}$, we converted their $\mathrm{U}-\mathrm{V}$ colour into a metallicity following the conversion of Reed, Harris \& Harris (1994), calibrated with GCs in M31. We used a foreground reddening value of $E(U-V)=0.2$ (Reed et al. 1994). Following Harris et al. (2004), Woodley et al. (2005) and Woodley et al. (2007), we divided our
GC sample into either metal-poor (when $[\mathrm{Fe} / \mathrm{H}]<-1.0$ ) or metal-rich (when $[\mathrm{Fe} / \mathrm{H}] \geq-1.0$ ). When neither $\mathrm{C}$, $\mathrm{T}_{1}, \mathrm{U}$ or $\mathrm{V}$ information was available, we assigned a GC as metal-rich if $(B-I) \geq 2.072$ and as metal-poor if $(B-I)<2.072$ following Peng et al. (2004b).

For the purpose of this study, we are interested in looking at the sizes of the most representative GCs, and so we have removed the GCs that deviate from the rest of the population in both ellipticity and half-light radii. There are 472 GCs that satisfy the requirements of having both ellipticity $<0.4$ and $r_{h}<8$ pc. We display the measured $r_{h}$ in Figure 6 as a function of galactocentric radius. The GCs are binned with effective galactic radius, $R_{\mathrm{eff}}$, of $\sim 5$ arcmin and fit each bin with a least squares fit. We note that within $1 R_{\text {eff }}$, the blue GCs do appear to be larger than the red, as has been seen in other GC systems, however, beyond 1-2 $R_{\text {eff }}$, this trend seems to disappear. In Figure 7, we separate the blue GCs from the red GCs and plot their half-light radii distributions as a function of the galaxy's effective radius. Along with the distributions, we show the mean $r_{h}$ in each bin. In the innermost bin, the mean halflight radius for the blue GCs is $\sim 30 \%$ larger than for the 


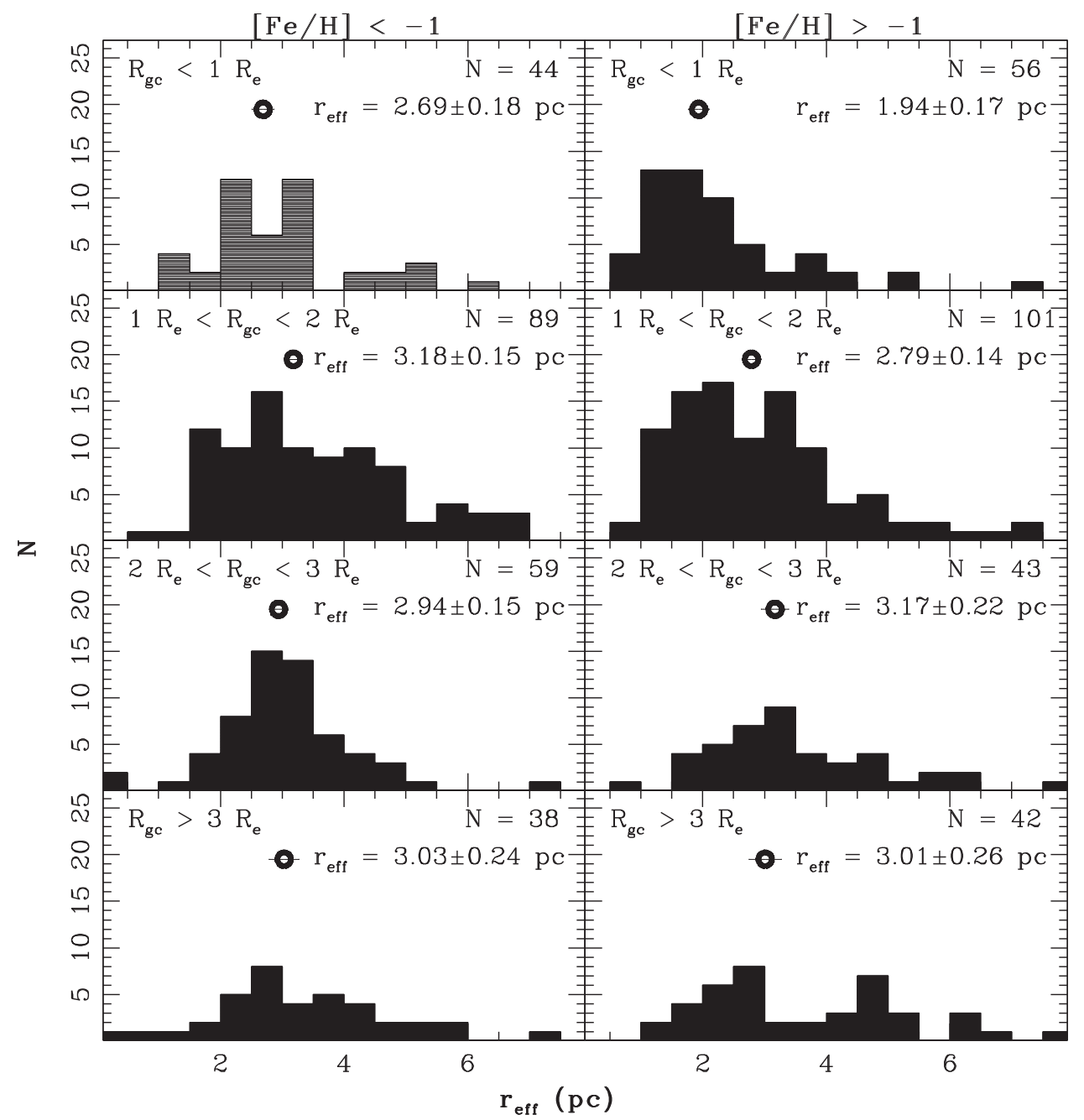

Figure 7 The distributions of the half-light radius for the blue (right) and red (left) GCs, as a function of effective radii in the galaxy. The mean half-light radii in each bin is plotted with uncertainty (circles).

red GCs. Beyond this distance the mean $r_{h}$ in each bin is not distinguishable between red and blue GCs. This is consistent with the results we found in Goméz \& Woodley (2007) for a much smaller dataset of GCs in NGC 5128.

\subsection{Structural Parameter Discussion}

The size difference between the red and blue GCs is very interesting and it is important to understand why the red GCs appear to be smaller in the inner regions of galaxies. There are a number of possible explanations for this.

One explanation suggests it could be caused by a projection effect (Larsen \& Brodie 2003). In the Milky Way, it has been found that the GCs follow a size-distance relation, such that the size of a GC is smaller towards the centre of the galaxy (van den Bergh, Morbey \& Pazder 1991). If GC systems in other galaxies follow this same trend, then they suggest that since the MR GCs are more centrally concentrated in most extralactic systems, they would appear in the inner regions to be smaller than the blue GCs. The more centrally concentrated red GCs would lie at smaller galactocentric distances in projection, thus have smaller radii. At larger distances, the projection would have less effect and the trend should dissappear. However, recent work by Harris (2009) who studied a large combined sample of GCs from massive galaxies out to a few effective radii in the galaxy, found the size difference between the red and blue GCs persisted at large distances.

The different sizes of GCs could be the combined effect of mass segregation and metallicity dependent stellar lifetimes (Jordán et al. 2005). This would yield a smaller $r_{h}$ for metal-rich GCs; however, you would expect this to be the case at any galactocentric radius.

The differences in sizes of blue and red GCs could be caused by the different tidal effects experienced during their formation (Harris 2009). In the centre of the galaxy, the potential well would be deeper than the outer regions, and the GCs may have been forced to form smaller in order to survive. This has been supported by recent work of Georgiev et al. $(2008,2009)$ who measured sizes for the GCs in the Large Magellanic Cloud and noticed they were larger than the normal GC population in the Milky Way. If the size difference in the inner regions of the galaxy is 
real, then this could suggest that metal-rich and metal-poor GCs in some extragalactic systems, did not form under the same conditions.

We require more homogeneous and large samples of GCs in a variety of galaxies in order to better understand what processes in $\mathrm{GC}$ formation or formation environment has on the formation sizes of GCs. Interestingly, the size differences of GCs may have a direct relation to the environment in which they form, allowing the possibility of GCs to probe formation history of their host galaxy in yet another way. We suggest that it is likely the combination of the environment that the GCs have formed with a projection effect that may be responsible for the size differences in the inner regions of the GCs within NGC 5128.

\section{Conclusions}

We have studied the GC system in NGC 5128 and presented their ages and metallicities, their kinematics, their structural parameters, and we have also used them as tracers to estimate the mass of NGC 5128.

We find, using a sample of $72 \mathrm{GCs}$, that the majority of metal-rich and metal-poor GCs are old with $68 \%$ having ages $>8 \mathrm{Gyr}$. We do find a small fraction with young ages $<5$ Gyr, all of which are metal-rich. These results suggest there could have been a number of formation epochs within NGC 5128, which resulted in the formation of a small portion of its stellar population.

Using over 560 GCs in NGC 5128, we performed a kinematic analysis, and find the metal-rich GCs have a mild rotation of $41 \pm 15 \mathrm{~km} \mathrm{~s}^{-1}$ and rotate around $191 \pm 18^{\circ} \mathrm{E}$ of $\mathrm{N}$, similar to the isophotal major axis of the galaxy. The metal-rich GCs have very mild rotation and do not appear to rotate around either isophotal major or minor axis. The velocity dispersion profile of the GCs is generally flat in the inner regions of the galaxy. Compared to the stellar halo population, we see a number of similarities to the metal-rich GCs, including the rotation axis and surface density profile of the PNe population, as well as approximate ages and metal enrichment of the stellar halo (Rejkuba et al. 2005). We also use the radial velocity measurements of the GCs to estimate a mass of NGC 5128 to be $5.5 \pm 1.9 \times 10^{11} \mathrm{M}_{\odot}$ and a mass-to-light ratio of $15.35 M_{\odot} / L_{B \odot}$ out to 20 arcmin.

We examine the structural parameters of a representative sample of normal GCs in NGC 5128 using superb IMACS images. We examine their half-light radii, and find within 1-2 effective radii of the galaxy, the blue GCs are $\sim 30 \%$ larger than the red GCs. Beyond this distance, however, the size difference seems to disappear. We suggest this may be the result of their environment of formation, combined with the projection effect.

The old ages and kinematics of the GCs within NGC 5128 suggests that the majority of GCs, and presumably stars, formed early on in a rapid, or perhaps multiple, collapse(s). This is supported by the old ages of the GCs and high $[\alpha / \mathrm{Fe}]$ for both metal-rich and metal-poor GCs. There is evidence for a large spread in metal-rich ages, indicating either minor merging or accretion of small neighbouring satellites in more recent times. However the fraction of young objects is heavily biased by our selection of targets. The youngest GCs that we find in our sample are a few Gyrs old.

\section{Acknowledgments}

K.A.W. would like to thank the science organizing committee for the opportunity to present this work at the Many Faces of Centaurus A Conference in Sydney as well as for financial support. M.G. thanks Proyecto DI-36-09/R for financial support.

\section{References}

Aarseth, S. J. \& Heggie, D. C., 1998, MNRAS, 297, 794

Barmby, P., Holland, S. \& Huchra, J. P., 2002, AJ, 123, 1937

Beasley, M. A., Bridges, T., Peng, E., Harris, W. E., Harris, G. L. H., Forbes, D. A. \& Mackie, G., 2008, MNRAS, 386, 1443

Bekki, K., Beasley, M. A., Brodie, J. P. \& Forbes, D. A., 2005, MNRAS, 363, 1211

Bergond, G., Zepf, S. E., Romanowsky, A. J., Sharples, R. M. \& Rhode, K. L., 2006, A\&A, 448, 155

Bridges, T., Gebhardt, K., Sharples, R., Faifer, F. R., Forte, J. C., Beasley, M. A., Zepf, S. E., Forbes, D. A., Hanes, D. A. \& Pierce, M., 2006, MNRAS, 373, 157

Burstein, D., Faber, S. M., Gaskell, C. M. \& Krumm, N., 1984, ApJ, 287,586

Carlson, M. N. et al., 1998, AJ, 115, 1778

Chien, L.-H., Barnes, J. E., Kewley, L. J. \& Chambers, K. C., 2007, ApJ, 660, 105

Côté, P. et al., 2001, ApJ, 559, 828

Côté, P., McLaughlin, D. E., Cohen, J. G. \& Blakeslee, J. P., 2003, ApJ,591,850

Dekel, A., Stoehr, F., Mamon, G. A., Cox, T. J., Novak, G. S. \& Primack, J. R., 2005, Natur, 437, 707

Dufour, R. J., Harvel, C. A., Martins, D. M., Schiffer, III, F. H., Talent, D. L., Wells, D. C., van den Bergh, S. \& Talbot, Jr., R. J., 1979, AJ, 84, 284

Evans, N. W., Wilkinson, M. I., Perrett, K. M. \& Bridges, T. J., 2003, ApJ, 583, 752

Georgiev, I. Y., Goudfrooij, P., Puzia, T. H. \& Hilker, M., 2008, AJ, 135,1858

Georgiev, I. Y., Puzia, T. H., Hilker, M. \& Goudfrooij, P., 2009, MNRAS, 392, 879

Goméz, M. \& Woodley, K. A., 2007, ApJ, 670, L105

Groudfrooij, P., Schweizer, F., Gilmore, D. \& Whitmore, B. C., 2007, AJ, 133, 2737

Harris, G. L. H., Geisler, D., Harris, H. C. \& Hesser, J. E., 1992, AJ, 104,613

Harris, W. E. \& Harris, G. L. H., 2002, AJ, 123, 3108

Harris, G. L. H., Harris, W. E., \& Geisler, D. 2004, AJ, 128, 723

Harris, W. E., Harris, G. L. H., Barmby, P., McLaughlin, D. E. \& Forbes, D. A., 2006, AJ, 132, 2187

Harris, W. E., 2009, ApJ, 699, 254

Hesser, J. E., Harris, H. C., van den Bergh, S. \& Harris, G. L. H., 1984, ApJ, 276, 491

Hesser, J. E., Harris, H. C., \& Harris, G. L. H., 1986, ApJL, 303, 51

Holtzman, J. A. et al., 1992, AJ, 103, 691

Hui, X., Ford, H. C., Freeman, K. C. \& Dopita, M. A., 1995, ApJ, 449,592

Hwang, H. S. et al., 2008, ApJ, 674, 869

Jordán, A. et al., 2005, ApJ, 634, 1002

Karachentsev, I. D. et al., 2002, A\&A, 385, 21

King, I. R., 1962, AJ, 67, 471

Kissler-Patig, M., Brodie, J. P., Schroder, L. L., Forbes, D. A., Grillmair, C. J. \& Huchra, J. P., 1998, AJ, 115, 105

Kundu, A. \& Whitmore, B. C., 1998, AJ, 116, 2841 
Kundu, A. \& Whitmore, B. C., 2001, AJ, 121, 2950

Kundu, A., Whitmore, B. C., Sparks, W. B., Macchetto, R. D., Zepf, S. E. \& Ashman, K. M., 1999, ApJ, 513, 733

Larsen, S. S., 1999, A\&ApS, 139, 393

Larsen, S. S., 2001, AJ, 122, 1782

Larsen, S. S. \& Brodie, J. P., 2003, ApJ, 593, 340

Larsen, S. S., Brodie, J. P., Huchra, J. P., Forbes, D. A. \& Grillmair, C. J., 2001a, AJ, 121, 2974

Larsen, S. S., Forbes, D. A. \& Brodie, J. P., 2001b, MNRAS, 327, 1116

Miller, B. W., Whitmore, B. C., Schweizer, F. \& Fall, S. M., 1997, AJ, 114, 2381

Peng, E. W., Ford, H. C. \& Freeman, K. C., 2004a, 602, 685

Peng, E. W., Ford, H. C. \& Freeman, K. C., 2004b, ApJ, 602, 705

Peng, E. W. et al., 2006, ApJ, 639, 95

Pierce, M. et al., 2006, MNRAS, 366, 1253

Puzia, T. H., Kissler-Patig, M., Brodie, J. P. \& Huchra, J. P., 1999, AJ, 118, 2734

Puzia, T. H., Zepf, S. E., Kissler-Patig, M., Hilker, M., Minniti, D. \& Goudfrooij, P., 2002, A\&A, 391, 453

Reed, L. G., Harris, G. L. H. \& Harris, W. E., 1994, AJ, 107, 555

Rejkuba, M., Greggio, L., Harris, W. E., Harris, G. L. H. \& Peng, E. W., 2005, 631, 262

Rejkuba, M., Dubath, P., Minniti, D. \& Meylan, G., 2007, A\&A, 469, 147

Richtler, T. et al., 2004, AJ, 127, 2094

Schiminovich, D., van Gorkom, J. H., van der Hulst, J. M. \& Kasow, S., 1994, ApJ, 423, L101

Schweizer, F. \& Seitzer, P., 1993, ApJ, 417, L29

Schweizer, F., Miller, B. W., Whitmore, B. C. \& Fall, S. M., 1996, AJ, 112, 1839

Schiavon, R. P., Rose, J. A., Courteau, S. \& MacArthur, L. A., 2005, ApJS, 160, 163

Schuberth, Y., Richtler, T., Dirsch, B., Hilker, M., Larsen, S. S., Kissler-Patig, M. \& Mebold, U., 2006, A\&A, 459, 391
Schweizer, F. \& Seitzer, P., 1998, AJ, 116, 2206

Spitler, L. R., Larsen, S. S., Strader, J., Brodie, J. P., Forbes, D. A \& Beasley, M. A., 2006, AJ, 132, 1593

Spitzer, L. J. \& Thuan, T. X., 1972, ApJ, 175, 31

Thomas, D., Maraston, C. \& Bender, R., 2003, MNRAS, 339, 897

Thomas, D., Maraston, C. \& Korn, A., 2004, MNRAS, 351, L19

Trager, S. C., Worthey, G., Faber, S. M., Burstein, D. \& Gonzalez, J. J., 1998, ApJS, 116, 1

Trancho, G., Bastian, N., Miller, B. W. \& Schweizer, F., 2007, ApJ, 664,284

White, S. D. M. \& Rees, M. J. 1978, MNRAS, 183, 341

van den Bergh, S., Hesser, J. E. \& Harris, G. L. H., 1981, AJ, 86, 24

van den Bergh, S., Morbey, C. \& Pazder, J., 1991, ApJ, 375, 594

West, M. J., Cótê, P., Marzke, R. O. \& Jordá, A., 2004, Natur, 427, 31

Whitmore, B. C., Schweizer, F., Leitherer, C., Borne, K. \& Robert, C., 1993, AJ, 106, 1354

Whitmore, B. C. \& Schweizer, F., 1995, AJ, 109, 960

Whitmore, B. C., Zhang, Q., Leitherer, C., Fall, S. M., Schweizer, F. \& Miller, B. W., 1999, AJ, 118, 1551

Woodley, K. A., Harris, W. E. \& Harris, G. L. H., 2005, AJ, 129, 2654

Woodley, K. A., Harris, W. E., Beasley, M. A., Peng, E. W., Bridges, T. J., Forbes, D. A. \& Harris, G. L. H., 2007, 134, 494

Woodley, K. A., Harris, W. E., Puzia, T. H., Gómez, M., Harris, G. L. H. \& Geisler, D., 2010, ApJ, 708, 1335

Woodley, K. A., Harris, W. E., Gómez, M., Geisler, D. \& Harris, G. L. H., 2009, AJ, submitted

Worthey, G. \& Ottaviani, D. L., 1997, ApJS, 111, 377

Worthey, G., Faber, S. M., Gonzalez, J. J. \& Burstein, D., 1994, ApJS, 94, 687

Zepf, S. E., Carter, D., Sharples, R. M. \& Ashman, K., 1995, ApJ, 445, L19

Zepf, S. E., Ashman, K. M., English, J., Freeman, K. C. \& Sharples, R. M., 1999, AJ, 118, 752 